\title{
Evaluation of Slug expression is useful for predicting lymph node metastasis and survival in patients with gastric cancer
}

Han Hee Lee ${ }^{1}$, Sung Hak Lee ${ }^{2^{*}}$ D, Kyo Young Song ${ }^{3^{*}}$, Sae Jung $\mathrm{Na}^{4}$, Joo Hyun $\mathrm{O}^{5}$, Jae Myung Park', Eun Sun Jung ${ }^{2}$, Myung-Gyu Choi ${ }^{1}$ and Cho Hyun Park ${ }^{6}$

\begin{abstract}
Background: Slug is a transcription factor that activates the epithelial-mesenchymal transition (EMT) process in cancer progression. The aim of our study was to evaluate the clinical significance of S/ug expression in gastric cancer.

Methods: The expression of Slug in gastric cancer tissues of 456 patients who underwent gastrectomy was evaluated by immunohistochemistry using tissue microarrays. Slug expression level was defined by the composite score determined by multiplying the tumor staining scores for intensity and extent. The associations of Slug expression with clinicopathological characteristics and overall and recurrence-free survival were analyzed.

Results: Patients were divided into three groups according to Slug composite score ( $\leq 4,6$, and 9). Low, mid, and high expression of Slug was observed in 104 (22.7\%), 130 (28.3\%), and 225 (49.0\%) of cases, respectively. Overall survival and recurrence-free survival progressively increased from high to low Slug expression. In terms of lymph node metastasis, the rate of positive lymph node metastasis was 38/104 (36.5\%), 79/130 (60.8\%), and 178/225 (79.1\%) in low, mid, and high Slug expression groups, respectively, displaying a tendency to increase with higher Slug expression. In a multivariate analysis adjusting for patient age, tumor size, tumor depth, and histology, high Slug expression was associated with a high rate of positive lymph node metastasis compared with low Slug expression (odds ratio 3.42; 95\% confidence interval, 1.74-6.69). In a subgroup analysis of $\mathrm{T} 1$ cancer, patients with negative Slug expression (defined as $<5 \%$ positive tumor cells or no/weak staining) showed no lymph node metastasis (0/13), whereas those with positive Slug expression showed $15.9 \%$ (17/107) lymph node metastasis, with a negative predictive value of $100 \%$.
\end{abstract}

Conclusions: High expression of Slug in gastric cancer tissue was associated with lymph node metastasis and poor survival. Evaluation of Slug would be useful for discriminating patients at high risk of lymph node metastasis in early gastric cancer.

Keywords: Slug, Gastric cancer, Epithelial-mesenchymal transition, Tissue microarray, Prognosis

\section{Background}

Gastric cancer is the third leading cause of cancer death worldwide, and almost 1 million new cases occur annually [1]. With the introduction of mass screening methods such as endoscopy and upper gastrointestinal series, the proportion of patients with early detection of

\footnotetext{
*Correspondence: hakjjang@catholic.ac.kr; skys9615@gmail.com

${ }^{2}$ Department of Hospital Pathology, Seoul St. Mary's Hospital, College of Medicine, The Catholic University of Korea, 222, Banpo-daero, Seocho-gu, Seoul 06591, Republic of Korea

${ }^{3}$ Division of Gastrointestinal Surgery, Department of Surgery, Uijeongbu St. Mary's Hospital, College of Medicine, The Catholic University of Korea, Seoul, Korea, 271, Cheonbo-ro Uijeongbu, Gyeonggi-do 480-717, Republic of Korea Full list of author information is available at the end of the article
}

early gastric cancer (EGC) or precancerous adenoma has been increasing $[2,3]$. Endoscopic submucosal dissection (ESD) has become the standard therapy for EGC because it is minimally invasive and allows en bloc and complete resection [4]. Recently, there has been an attempt to expand the indications of ESD [5]. Along with this, prediction of lymph node metastasis (LNM) in EGC is becoming more important because LNM is one of the most important factors for assessment of prognosis and decision of therapeutic modalities [6, 7]. Advanced gastric cancer (AGC) has a particularly poor prognosis compared with EGC. AGC spreads locally by breaking through the gastric wall into neighboring tissue 
and metastasizes to regional lymph nodes. The presence of metastatic lymph nodes could be an outstanding prognostic factor. Differences in the prognoses of patients with negative lymph node metastasis versus positive lymph node metastasis are especially robust in surgically treated AGC [8-10].

Epithelial-mesenchymal transition (EMT) is a biologic process by which epithelial cells lose their cell-cell junctions and apical-basal polarity and gain a highly motile and invasive phenotype to become mesenchymal cells [11]. EMT is integral to embryo formation and organ development [12] and has also been shown to occur during wound healing and tissue fibrosis [13]. In cancer, EMT contributes pathologically to cancer progression by enabling primary tumor cells to break through the basal lamina and invade adjacent tissue, leading to tumor metastasis [14].

Slug, also known as Snail2, is one of the key transcription factors that activate EMT process in cancer progression [15]. It contributes to repression of the epithelial phonotype by binding to E-box DNA sequences in the proximal promoter region of the E-cadherin gene [16-18]. This role as a strong E-cadherin repressor mediates loss of tight junctions of epithelial cells and initiates EMT, which facilitates cancer cell invasion and distant metastasis [18, 19]. Slug has been highly studied in various cancers. In breast cancer patients, Slug is consistently overexpressed in aggressive and basal-type breast tumors [20] and seems to be involved in breast tumorigenesis and metastasis through regulation of the EMT [21]. It has also been demonstrated that Slug expression is correlated with poor prognosis in pancreatic and esophageal cancer patients [22, 23]. Recent studies have revealed that Slug not only functions in cancer metastasis, but also plays a role in cancer stemness [24, 25], implying that Slug participates in early steps of cancer progression.

In gastric cancer, upregulation of Slug mRNA is associated with suppression of E-cadherin in intestinal and diffuse type gastric carcinomas [26]. In a study focused on protein expression, high Slug expression was correlated with advanced stages and worse clinical outcomes [27]. However, there are only a few studies on the clinical significance of Slug in gastric cancer. In addition, the significance of Slug expression in early gastric cancer has not been proved.

Therefore, the purpose of our study was to evaluate the clinical significance of Slug expression in gastric cancer using a tissue microarray method in a large series of patients with resected gastric cancer.

\section{Methods}

\section{Patients and clinical samples}

A total of 459 patients (313 men and 146 women) were randomly selected by random number generation from
2495 consecutive patients with gastric cancer who had undergone radical surgery at Seoul St. Mary's Hospital, The Catholic University of Korea, between 2000 and 2009. Clinicopathological data were reviewed retrospectively from the participants' medical records and pathology reports at our institution. Variable factors including age, gender, type of surgery, tumor size, location, pathologic staging, histology, and lymphatic, venous, and perineural invasion were analyzed. Tumor location was categorized into upper, middle, and lower thirds of the stomach. The gastric cancers were staged according to the pathological tumor/node/metastasis (pTNM) classification (8th edition) of the Union for International Cancer Control [28]. The histological types of the gastric cancers were assessed according to the 2010 World Health Organization classification [29]. Tumors were also classified into intestinal, diffuse, and mixed types by Lauren classification [30]. Written informed consent was obtained from all patients. Patient consent and specimen collection were conducted in accordance with protocols approved by the Institutional Review Board of The Catholic University of Korea (KC14SISI0158).

\section{Tissue microarray construction and immunohistochemistry}

All gastric specimens were histologically reviewed, and tissue microarrays (TMAs) were constructed from each of the formalin-fixed, paraffin-embedded (FFPE) tissue blocks using a Manual Tissue Arrayer (Beecher Instruments, Sun Prairie, WI, USA) with a $2.0-\mathrm{mm}$ tip.

Immunohistochemical analysis was performed using primary antibody against Slug (ab188875) (polyclonal; 1:150; Abcam, Cambridge, UK). We determined the optimal dilution of the Slug antibody using positive control tissue such as normal gastric epithelial cells and placenta. Four-micrometer-thick tissue sections from the TMA blocks were transferred to Probe On Plus slides (Fisher Scientific, Pittsburgh, PA, USA) and baked for $2 \mathrm{~h}$ in a dry oven at $56{ }^{\circ} \mathrm{C}$ (Agilent Technologies, Santa Clara, CA, USA). The FFPE sections were deparaffinized in xylene three times and rehydrated through $100 \%, 90 \%$, $80 \%$, and $70 \%$ ethanol in Tris-buffered saline $(\mathrm{pH} 7.4$ ). Antigen retrieval was achieved by boiling in $10 \mathrm{mM}$ sodium citrate buffer ( $\mathrm{pH}$ 6.0) using a microwave oven for 20 min. After treatment with $3 \% \mathrm{H}_{2} \mathrm{O}_{2}$ in phosphatebuffered saline, the tissues were incubated with primary antibody at $4{ }^{\circ} \mathrm{C}$ overnight and then with diluted (1:100) biotinylated anti-mouse antibody (Abnova, Walnut, CA, USA) for $1 \mathrm{~h}$ at room temperature. The signal was amplified using diluted ExtrAvidin-peroxidase (1:50; SigmaAldrich, St. Louis, MO, USA) for $1 \mathrm{~h}$ at room temperature and visualized using the liquid 3,3'-diaminobenzidine + Substrate Chromogen system (Dako, Glostrup, Denmark). Counterstaining was performed with hematoxylin. 
Nonspecific staining was not observed in any negative control sections.

\section{Evaluation of immunohistochemical staining}

Two pathologists (SH Lee and ES Jung) who were blinded to the clinicopathological parameters independently reviewed the immunohistochemical staining for the tissue sections. We used a semi-quantitative scoring system based on the intensity and extent of stained cells for each case. The staining intensity was graded from 0 to $3(0=$ no expression at all, $1=$ weak, $2=$ moderate, $3=$ strong). The extent was graded from 0 to 3 $(0=<5 \%, 1=5-25 \%, 2=26-50 \%, 3=>50 \%)$. The intensity scores and extent scores were multiplied to obtain the composite score.

\section{Statistical analysis}

Continuous data are presented as mean \pm standard deviation, and categorical data are presented as quantity and proportion. Pearson's $\chi^{2}$ test for categorical variables and Student's $t$ test for unpaired data for continuous variables were performed to compare clinicopathological characteristics among the three Slug expression groups. A $P$ value $<0.05$ was considered significant. Survival rates were calculated by the Kaplan-Meier method, with the date of gastrectomy as the starting point. Patients who were alive were censored at the time of the last follow-up. Differences in survival were examined by the log-rank test. Multivariable analysis was performed using a Cox proportional hazards model with a backward stepwise selection procedure. All analyses were performed by SAS for Windows software (version 8.02, SAS Institute, Cary, NC, USA).

\section{Results}

\section{Expression profile of Slug in gastric cancer}

Table 1 shows overall immunohistochemical Slug expression in the gastric cancer tissue microarray. More than half of the tissues showed diffuse Slug expression, which corresponds to extent score 3, and 71.9\% (330/ 459) of tissues showed intensity score 3 , indicating strong staining. Figure 1 shows representative images of the range of Slug staining intensity. Multiplication of these two variables yielded the Slug composite score, which ranged from 0 to 9 . Classification of the patients according to Slug composite score yielded $104(22.7 \%)$, 130 (28.3\%), and $225(49.0 \%)$ patients in the low, mid, and high Slug groups, respectively.

\section{Relationships between Slug expression and clinicopathological parameters}

Table 2 summarizes the clinicopathological characteristics of the 459 patients undergoing gastrectomy for gastric cancer. The mean age of the patients was 58.6 years
Table 1 Scoring methods of Slug expression

\begin{tabular}{|c|c|c|}
\hline Measures & Number & Percent \\
\hline \multicolumn{3}{|l|}{ Extent } \\
\hline 0 : negative $(<5 \%)$ & 15 & 3.3 \\
\hline 1: sporadic (5-25\%) & 35 & 7.6 \\
\hline 2: focal (25-50\%) & 141 & 30.7 \\
\hline 3: diffuse (>50\%) & 268 & 58.4 \\
\hline \multicolumn{3}{|l|}{ Intensity } \\
\hline 0: no staining & 2 & 0.4 \\
\hline 1: weak staining & 24 & 5.2 \\
\hline 2: moderate staining & 103 & 22.4 \\
\hline 3: strong staining & 330 & 71.9 \\
\hline \multicolumn{3}{|l|}{ Extent $\times$ Intensity } \\
\hline \multicolumn{3}{|c|}{$=$ Slug composite score (range 0-9) } \\
\hline $\operatorname{Low}(\leq 4)$ & 104 & 22.7 \\
\hline Mid (6) & 130 & 28.3 \\
\hline High (9) & 225 & 49.0 \\
\hline
\end{tabular}

(range $23-86$ years), and $68.2 \%(n=313)$ were male. Distal subtotal gastrectomy was the most commonly performed surgery (63.2\%). The high Slug group tended to have large tumors and advanced tumor depth and stages. They also had a high rate of positive perineural invasion. Regarding histology, the proportion of poorly differentiated adenocarcinoma tended to increase from low to high Slug expression groups. However, the proportion of signet ring cell carcinoma was highest in the low Slug group.

\section{Slug expression and lymph node metastasis}

The rate of positive lymph node metastasis was $36.5 \%$ in the low group, $60.8 \%$ in the mid group, and $79.1 \%$ in the high Slug expression group, thus displaying a tendency to increase with increasing Slug expression (Table 3). Positive lymph node ratio calculated by dividing number of metastatic LNs by number of retrieved LNs was significantly higher in the high Slug group. The high Slug group also showed a high proportion of positive lymphatic invasion.

In a multivariate logistic regression analysis for lymph node metastasis, Slug composite score was identified as an independent predictive factor for lymph node metastasis even after adjusting for age, tumor size, tumor depth, and Lauren classification (Table 4). Compared with patients with low Slug score, the adjusted odds ratio in the high Slug group was 3.42 (95\% confidence inter$\mathrm{val}=1.74-6.69)$. Tumor size and depth were also identified as predictive factors for lymph node metastasis.

The recurrence rates of gastric cancer were compared between the three Slug groups (Fig. 2). Patients with high Slug score had the highest tumor recurrence rate. 

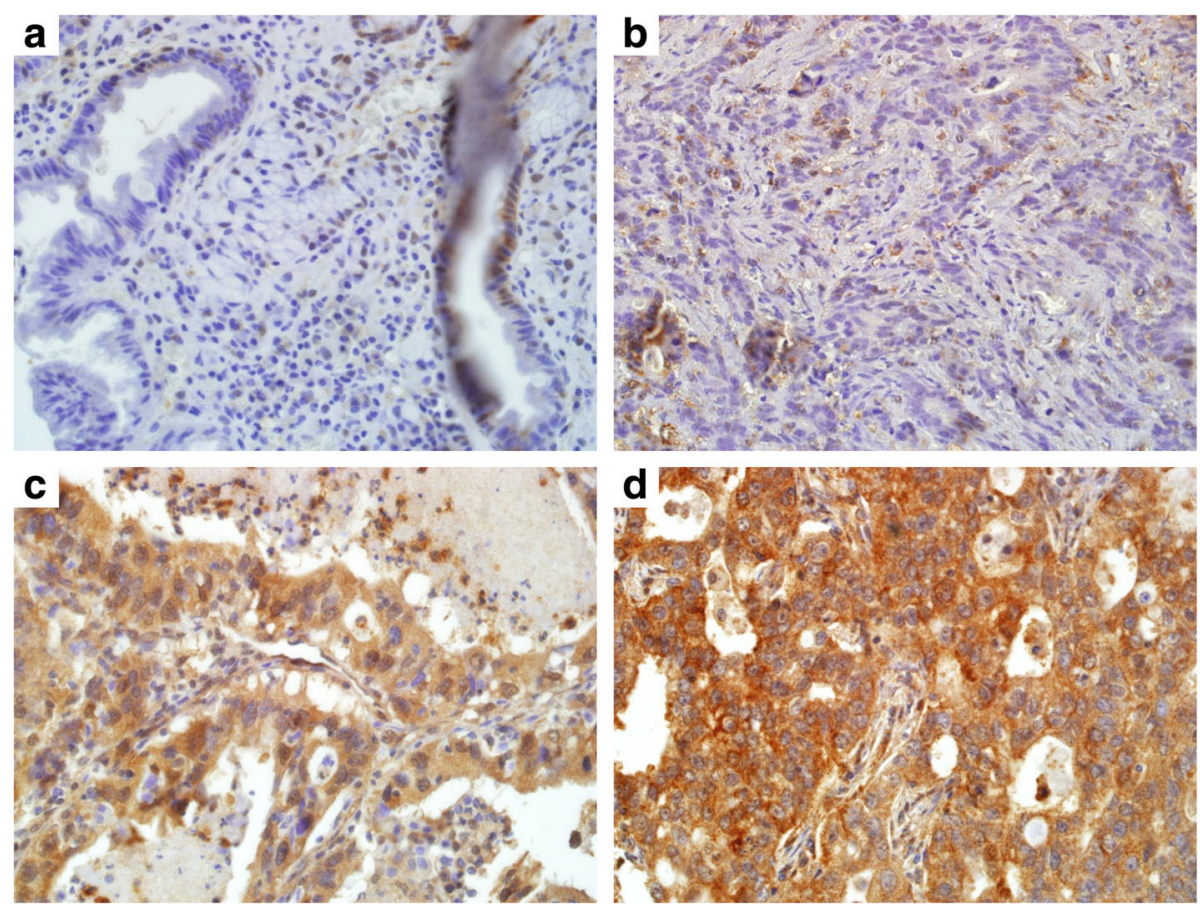

Fig. 1 Immunohistochemistry findings showing expression of Slug in gastric cancer tissue. a no staining. b weak staining. c moderate staining d strong staining

The rate of recurrence was significantly higher in the high Slug group than in the low $(P<0.001)$ and mid $(P=0.006)$ Slug groups. There was no statistically significant difference between the low and mid Slug groups $(P=0.280)$.

\section{Slug expression and survival}

Overall survival rates were determined with respect to the Slug composite score using the log rank test (Fig. 3). The 5-year overall survival rate was significantly worse in the high Slug group compared with the mid $(61.5 \%$ versus $72.4 \% ; P=0.017)$ and low $(61.5 \%$ versus $84.6 \%$; $\mathrm{P}<0.001)$ Slug groups. The low Slug group had the best 5 -year overall survival rate. In multivariable Cox regression analysis including age, gender, TNM stage, Lauren classification, and Slug composite score, Slug score was not significantly associated with overall survival, whereas age and TNM stage remained independent prognostic factors (Additional file 1: Table S1).

\section{Subgroup analysis of T1 tumors}

We conducted a subgroup analysis of T1 tumors (Table 5). Negative Slug expression was defined as $<5 \%$ positive tumor cells or no/weak staining intensity. Tumor depth and size were not significantly different between negative and positive Slug expression. Approximately $60 \%$ of cases with negative Slug expression were signet ring cell carcinoma.
The rate of lymph node metastasis in T1 tumor was $14.2 \%(17 / 120)$. Patients with negative Slug expression showed no lymph node metastasis (0/13), whereas those with positive Slug expression showed $15.9 \%(17 / 107)$ lymph node metastasis, with a negative predictive value of $100 \%$.

\section{Discussion}

The present study aimed to determine the relationship between Slug expression and prognosis in patients with gastric cancer. High Slug expression according to our composite score was observed in about $50 \%$ of gastric cancer tissues. We demonstrated that the expression of Slug is associated with tumor progression and poor prognosis in gastric cancer. Especially, Slug expression was highly correlated with various indicators reflecting lymphatic progression such as lymph node metastasis, lymphatic invasion, and positive lymph node ratio. As it is reasonable to consider that advanced cancer has greater migrating activity and invasiveness than EGC, this finding supports the hypothesis that Slug, one of the important EMT drivers, is involved in lymphatic metastasis of gastric cancer through the EMT process. In the case of T1 tumor confirmed after surgical resection, negative Slug expression might exclude lymph node metastasis of EGC.

To the best of our knowledge, there is only one previous study that investigated Slug protein expression in 
Table 2 Comparison of characteristics of the patients according to Slug composite score

\begin{tabular}{|c|c|c|c|c|c|}
\hline Measures & Total patients $(N=459)$ & $\operatorname{Low}(n=104)$ & Mid $(n=130)$ & High $(n=225)$ & $P$ \\
\hline \multicolumn{6}{|l|}{ Age (years) } \\
\hline Mean \pm SD & $58.6 \pm 11.9$ & $56.3 \pm 12.3$ & $58.9 \pm 11.8$ & $59.6 \pm 11.6$ & 0.064 \\
\hline Range & $23-86$ & $23-81$ & $32-82$ & $24-86$ & \\
\hline Male & $313(68.2 \%)$ & $65(62.5 \%)$ & $94(72.3 \%)$ & $154(68.4 \%)$ & 0.276 \\
\hline \multicolumn{6}{|l|}{ Type of surgery } \\
\hline Total gastrectomy & $166(36.2 \%)$ & $30(28.8 \%)$ & $51(39.2 \%)$ & $85(37.8 \%)$ & \\
\hline Subtotal gastrectomy & $290(63.2 \%)$ & $73(70.2 \%)$ & $78(60.0 \%)$ & $139(61.8 \%)$ & \\
\hline Wedge resection & $3(0.7 \%)$ & $1(1.0 \%)$ & $1(0.8 \%)$ & $1(0.4 \%)$ & \\
\hline \multicolumn{6}{|l|}{ Tumor size (cm) } \\
\hline Mean \pm SD & $5.0 \pm 2.9$ & $4.1 \pm 2.5$ & $5.1 \pm 3.3$ & $5.4 \pm 2.8$ & 0.001 \\
\hline Range & $0.2-19.0$ & $0.4-12.5$ & $0.5-19.0$ & $0.2-15.5$ & \\
\hline \multicolumn{6}{|l|}{ Location } \\
\hline Upper third & $80(17.4 \%)$ & $16(15.4 \%)$ & $22(16.9 \%)$ & $42(18.7 \%)$ & 0.599 \\
\hline Middle third & $164(35.7 \%)$ & $43(41.3 \%)$ & $50(38.5 \%)$ & $71(31.6 \%)$ & \\
\hline Lower third & 206 (44.9\%) & 44 (42.3\%) & $55(42.3 \%)$ & $107(47.6 \%)$ & \\
\hline Whole stomach & $9(2.0 \%)$ & $1(1.0 \%)$ & $3(2.3 \%)$ & $5(2.2 \%)$ & \\
\hline \multicolumn{6}{|l|}{ Tumor depth (pT) } \\
\hline T1 & $120(26.1 \%)$ & $56(53.8 \%)$ & $30(23.1 \%)$ & $34(15.1 \%)$ & $<0.001$ \\
\hline $\mathrm{T} 2$ & $62(13.5 \%)$ & $18(17.3 \%)$ & $26(20.0 \%)$ & $18(8.0 \%)$ & \\
\hline T3 & $121(26.4 \%)$ & $14(13.5 \%)$ & 35 (26.9\%) & $72(32.0 \%)$ & \\
\hline T4 & $156(34.0 \%)$ & $16(15.4 \%)$ & 39 (30.0\%) & $101(44.9 \%)$ & \\
\hline \multicolumn{6}{|l|}{ TNM Stage } \\
\hline I & $131(28.5 \%)$ & $62(59.6 \%)$ & 37 (28.5\%) & $32(14.2 \%)$ & $<0.001$ \\
\hline$\|$ & $122(26.6 \%)$ & $25(24.0 \%)$ & $45(34.6 \%)$ & $52(23.1 \%)$ & \\
\hline III & $206(44.9 \%)$ & $17(16.3 \%)$ & 48 (36.9\%) & $141(62.7 \%)$ & \\
\hline \multicolumn{6}{|l|}{ Venous invasion ${ }^{a}$} \\
\hline Negative & $406(88.5 \%)$ & 97 (94.2\%) & $117(90.0 \%)$ & $192(85.3 \%)$ & 0.055 \\
\hline Positive & $52(11.3 \%)$ & $6(5.8 \%)$ & $13(10.0 \%)$ & $33(14.7 \%)$ & \\
\hline \multicolumn{6}{|l|}{ Perineural invasion } \\
\hline Negative & $270(58.8 \%)$ & 81 (77.9\%) & 75 (57.7\%) & $114(50.7 \%)$ & $<0.001$ \\
\hline Positive & $189(41.2 \%)$ & $23(22.1 \%)$ & $55(42.3 \%)$ & $111(49.3 \%)$ & \\
\hline \multicolumn{6}{|l|}{ Histology } \\
\hline Adenocarcinoma & & & & & $0.005^{b}$ \\
\hline Well differentiated & $38(8.3 \%)$ & $12(11.5 \%)$ & $5(3.8 \%)$ & $21(9.3 \%)$ & \\
\hline Moderately differentiated & $136(29.6 \%)$ & $23(22.1 \%)$ & $44(33.8 \%)$ & $69(30.7 \%)$ & \\
\hline Poorly differentiated & $189(41.2 \%)$ & 35 (33.7\%) & $53(40.8 \%)$ & $101(44.9 \%)$ & \\
\hline Mucinous adenocarcinoma & $19(4.1 \%)$ & $5(4.8 \%)$ & $7(5.4 \%)$ & 7 (3.1\%) & \\
\hline Signet ring cell carcinoma & $77(16.8 \%)$ & 29 (27.9\%) & $21(16.2 \%)$ & 27 (12.0\%) & \\
\hline \multicolumn{6}{|l|}{ Lauren classification } \\
\hline Intestinal & $174(37.9 \%)$ & 40 (38.5\%) & 47 (36.2\%) & 87 (38.7\%) & 0.433 \\
\hline Diffuse & 177 (38.6\%) & 40 (38.5\%) & $58(44.6 \%)$ & 79 (35.1\%) & \\
\hline Mixed & $108(23.5 \%)$ & 24 (23.1\%) & 25 (19.2\%) & 59 (26.2\%) & \\
\hline
\end{tabular}

Where appropriate, data are shown as the mean \pm SD

a Lymphatic and venous invasion could not be evaluated in 2 and 1 cases, respectively

binear-by-linear association 
Table 3 Association of lymphatic metastasis and Slug expression

\begin{tabular}{|c|c|c|c|c|c|}
\hline Measures & Total patients $(N=459)$ & Low $(n=104)$ & Mid $(n=130)$ & High $(n=225)$ & P \\
\hline \multicolumn{6}{|l|}{ Lymph node metastasis (pN) } \\
\hline Negative & $164(35.7 \%)$ & $66(63.5 \%)$ & $51(39.2 \%)$ & $47(20.9 \%)$ & \multirow[t]{7}{*}{$<0.001$} \\
\hline Positive & $295(64.3 \%)$ & $38(36.5 \%)$ & $79(60.8 \%)$ & $178(79.1 \%)$ & \\
\hline N1 & $98(21.4 \%)$ & $20(19.2 \%)$ & $30(23.1 \%)$ & $48(21.3 \%)$ & \\
\hline N2 & $99(21.6 \%)$ & $15(14.4 \%)$ & $26(20.0 \%)$ & $58(25.8 \%)$ & \\
\hline N3 & $98(21.4 \%)$ & $3(2.9 \%)$ & $23(17.7 \%)$ & $72(32.0 \%)$ & \\
\hline N3a & $94(20.5 \%)$ & $3(2.9 \%)$ & $20(15.4 \%)$ & $71(31.6 \%)$ & \\
\hline $\mathrm{N} 3 \mathrm{~b}$ & $4(0.9 \%)$ & $0(0.0 \%)$ & $3(2.3 \%)$ & $1(0.4 \%)$ & \\
\hline Number of metastatic lymph nodes & $3.7 \pm 4.9(0-42)$ & $1.2 \pm 2.3(0-12)$ & $3.6 \pm 5.8(0-42)$ & $4.9 \pm 4.7(0-25)$ & $<0.001$ \\
\hline Number of retrieved lymph nodes & $42.4 \pm 15.4(6-106)$ & $39.5 \pm 13.3(14-78)$ & $44.9 \pm 16.1^{*}(8-97)$ & $42.4 \pm 15.8(6-106)$ & 0.028 \\
\hline Positive lymph node ratio & $0.09 \pm 0.12$ & $0.03 \pm 0.06$ & $0.08 \pm 0.12$ & $0.12 \pm 0.13$ & $<0.001$ \\
\hline \multicolumn{6}{|l|}{ Lymphatic invasion ${ }^{a}$} \\
\hline Negative & $154(33.6 \%)$ & $59(57.3 \%)$ & 45 (34.9\%) & $50(22.2 \%)$ & \multirow[t]{2}{*}{$<0.001$} \\
\hline Positive & $303(66.0 \%)$ & $44(42.7 \%)$ & $84(65.1 \%)$ & $175(77.8 \%)$ & \\
\hline
\end{tabular}

" $p<0.05$; when compared with "low Slug composite score group" using the ANOVA test with post-hoc Tukey-HSD test

aymphatic invasion could not be evaluated in 2 cases

gastric cancer tissues by immunohistochemical staining [27]. In that study, 30\% of tissues showed positive Slug expression, defined as detectable immunoreaction in the perinuclear and other cytoplasmic regions of more than $10 \%$ of the cancer cells. This is in contrast to findings from the current study showing that about $75 \%$ of gastric cancer patients had mid to high Slug expression. A possible explanation for this finding is that many more advanced cancers were included in our study compared to the previous study; approximately $60 \%$ of patients in the previous study were stage I, compared with only about 30\% in our study. In another previous study based on mRNA expression of Slug by real-time quantitative RT-PCR, 58\% of gastric cancer patients showed Slug upregulation in the tumor, which is in close agreement with our finding [26]. Moreover, the tendency for Slug expression to be associated with advanced pTNM stages was observed in both studies [26, 27]. The correlation of Slug expression with increased tumor size and perineural invasion was newly identified in the present study.

We focused on the association of lymphatic metastasis and Slug expression because Slug can activate the EMT

Table 4 Multivariate analysis showing independence of the effect on lymph node metastasis

\begin{tabular}{|c|c|c|c|c|}
\hline & Number of patients & Odds ratio & $95 \% \mathrm{Cl}$ & $P$ \\
\hline Age & & 1.01 & $0.99-1.04$ & 0.277 \\
\hline Tumor size & & 1.12 & $1.00-1.25$ & 0.049 \\
\hline \multicolumn{5}{|l|}{ Tumor depth (pT) } \\
\hline $\mathrm{T} 1$ & $120(26.1 \%)$ & 1 (ref) & & \\
\hline $\mathrm{T} 2$ & $62(13.5 \%)$ & 17.14 & $7.70-38.17$ & $<0.001$ \\
\hline T3 & $121(26.4 \%)$ & 33.99 & $14.87-77.71$ & $<0.001$ \\
\hline T4 & $156(34.0 \%)$ & 13.35 & $6.22-28.64$ & $<0.001$ \\
\hline \multicolumn{5}{|l|}{ Lauren classification } \\
\hline Intestinal & 174 (37.9\%) & 1 (ref) & & \\
\hline Diffuse + Mixed & $285(62.1 \%)$ & 1.07 & $0.61-1.88$ & 0.825 \\
\hline \multicolumn{5}{|c|}{ Slug composite score } \\
\hline Low & $104(22.7 \%)$ & 1 (ref) & $1.09-1.76$ & \\
\hline Mid & $130(28.3 \%)$ & 1.33 & $0.67-2.63$ & 0.413 \\
\hline High & 225 (49.0\%) & 3.42 & $1.74-6.69$ & $<0.001$ \\
\hline
\end{tabular}




\section{Recurrence rate by S/ug expression}

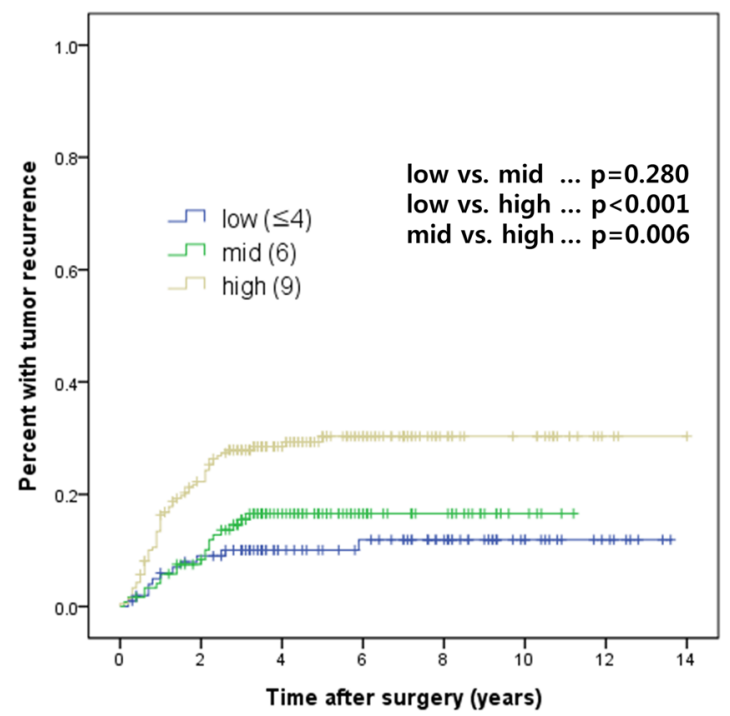

Fig. 2 Cumulative recurrence rates according to Slug expression after gastrectomy

process. For this purpose, we used the Slug composite score to produce a more continuous scale (low, mid, and high Slug groups) instead of dichotomizing the patient groups. As expected, higher Slug expression was associated with more prevalent lymph node metastasis and lymphatic invasion. In addition, the positive lymph node ratio gradually increased with increasing Slug score. This ratio represents lymph node metastasis density [31]. Much study has focused on this ratio because it has global

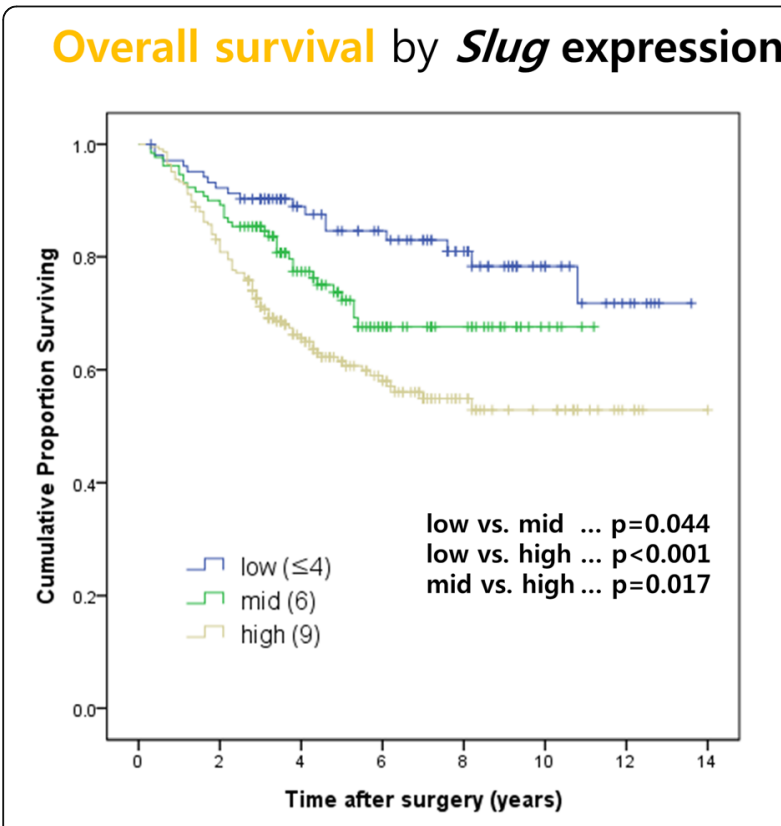

Fig. 3 Overall survival according to Slug expression after gastrectomy prognostic relevance in gastric cancer regardless of stage in multivariable analysis and is more sophisticated than conventional nodal metastasis in TNM staging for predicting prognosis [32]. In addition, we demonstrated that Slug expression is an independent prognostic factor for lymph node metastasis in gastric cancer patients even after adjustment for well-known prognostic factors including tumor size and depth of tumor invasion.

The current study indicates that Slug expression correlates well with overall survival as well as tumor recurrence. The high Slug expression group had the worst long-term survival rate and the highest tumor recurrence rate. These results correspond well with previous studies, in which positive Slug expression was associated with distant metastasis and poor postoperative 5-year survival [26, 27]. To our knowledge, this is the first report of long-term survival and recurrence data according to Slug expression and suggests that EMT signaling with involvement of Slug could affect long-term prognosis after gastrectomy of gastric cancer patients.

In a subgroup analysis of T1 tumors, we documented that Slug expression is associated with unexpected lymph node metastasis in EGC. EGC is defined as gastric cancer that invades no more deeply than the submucosa, irrespective of lymph node metastasis [33]. It has been reported that about $10-15 \%$ of patients with EGC have lymph node metastasis [1, 34, 35]. Precise prediction of lymph node metastasis status in EGC is a very important issue because ESD has become increasingly popular as a minimally invasive treatment for EGC [36]. We applied strict criteria for negative Slug expression in order to increase the negative predictive value because false negative results could be fatal when making the decision between surgical resection and ESD. In our study, all patients with T1 tumor and negative Slug expression showed no lymph node metastasis even though some of them had submucosal tumor invasion (T1b) or undifferentiated (poorly differentiated or signet ring cell) type histology. Tumor depth beyond submucosa and histological differentiation are well known independent risk factors for lymph node metastasis of EGC [37, 38]. Interestingly, 8 of 13 Slug negative T1 tumors were signet ring cell cancer. A previous study showed that signet ring foci of 8 patients with hereditary diffuse gastric cancer had a low proliferative index and there was no evidence for EMT [39]. This finding corresponds well with our result.

Our study has some strengths. First, a relatively large number of patients were randomly selected from consecutive patients undergoing surgery for gastric cancer for TMA and analyzed. Moreover, we present a novel finding regarding greater than 5-year survival and tumor recurrence according to Slug expression. In addition, this is the first report to document the significance of Slug expression in EGC. 
Table 5 Subgroup analysis of T1 tumor according to Slug expression

\begin{tabular}{|c|c|c|c|c|}
\hline & \multirow[t]{2}{*}{ Total } & \multicolumn{2}{|l|}{ Slug expression } & \multirow[t]{2}{*}{$P$} \\
\hline & & Negative $(n=13)$ & Positive $(n=107)$ & \\
\hline \multicolumn{5}{|l|}{ Tumor depth } \\
\hline T1a & 56 & $6(46.2 \%)$ & 50 (46.7\%) & \multirow[t]{2}{*}{0.969} \\
\hline $\mathrm{T} 1 \mathrm{~b}$ & 64 & $7(53.8 \%)$ & 57 (53.5\%) & \\
\hline Tumor size & $3.0 \pm 1.9$ & $2.7 \pm 2.0$ & $3.0 \pm 1.9$ & 0.670 \\
\hline \multicolumn{5}{|l|}{ Histology } \\
\hline Adenocarcinoma, WD & 25 & $0(0.0 \%)$ & 25 (23.4\%) & \multirow[t]{4}{*}{$0.001^{a}$} \\
\hline Adenocarcinoma, MD & 37 & $3(23.1 \%)$ & $34(31.8 \%)$ & \\
\hline Adenocarcinoma, PD & 31 & $2(15.4 \%)$ & $29(27.1 \%)$ & \\
\hline Signet ring cell cancer & 27 & $8(61.5 \%)$ & 19 (17.8\%) & \\
\hline \multicolumn{5}{|l|}{ Lymph node metastasis } \\
\hline Negative & 103 & $13(100 \%)$ & $90(84.1 \%)$ & \multirow[t]{2}{*}{$0.210^{b}$} \\
\hline Positive & 17 & $0(0.0 \%)$ & $17(15.9 \%)$ & \\
\hline
\end{tabular}

WD well differentiated, $M D$ moderately differentiated, $P D$ poorly differentiated

aLinear-by-linear association

${ }^{\mathrm{b}}$ Fisher's exact test

\section{Conclusions}

Our data demonstrated that high expression of Slug in gastric cancer tissue was associated with higher tumor recurrence rate and poor long-term survival. In particular, in cases with lymph node metastasis Slug expression was an independent predictive factor regardless of tumor size or depth of tumor invasion. Negative Slug expression showed high negative predictive value for lymph node metastasis in EGC, which could have potential for future use in discriminating patients with EGC at high risk of lymph node metastasis.

\section{Additional file}

Additional file 1: Table S1. Multivariate analysis showing independence of the effect on overall mortality. (DOCX $15 \mathrm{~kb}$ )

\section{Abbreviations}

AGC: Advanced gastric cancer; EGC: Early gastric cancer; EMT: Epithelialmesenchymal transition; ESD: Endoscopic submucosal dissection; FFPE: Formalin-fixed, paraffin-embedded; LNM: Lymph node metastasis; pTNM: Pathological tumor/node/metastasis; TMAs: Tissue microarrays

\section{Acknowledgements}

Not applicable

\section{Funding}

This study was supported by Research Fund of College of Medicine, The Catholic University of Korea, by a program of Catholic Medical Center Research Foundation made in 2014, and by the National Research Foundation of Korea (NRF) grant funded by the Korea government (Ministry of Science, ICT \& Future Planning) (NRF-2016R1C1B2015185).

\section{Availability of data and materials}

Attributing to the privacy of patients, the patient information is publicly inaccessible.

\section{Authors' contributions}

HHL participated in the study design, performed the data collection and chart review, and drafted the manuscript with help of other authors. SHL participated in planning the study, performed histological examination of the samples, evaluated IHC results and helped finalize the manuscript. KYS designed the treatment protocol, performed the surgeries, and helped finalize the manuscript. SJN and $\mathrm{JHO}$ participated in the study design. JMP participated in the study design and helped finalize the manuscript. ESJ performed the pathological studies and made pathology pictures. MGC participated in planning the study and helped to gather clinical data. CHP participated in planning the study and performed the surgeries. All authors have read and approved the final version of this manuscript.

\section{Ethics approval and consent to participate}

The study with human samples was approved by the Ethics Committee of the Catholic University of Korea (KC14SISI0158). We clarify that all clinical samples described here were gained from patients who had given written informed consent.

\section{Consent for publication}

Not applicable

\section{Competing interests}

The authors declare that they have no competing interests.

\section{Publisher's Note}

Springer Nature remains neutral with regard to jurisdictional claims in published maps and institutional affiliations.

\footnotetext{
Author details

'Department of Internal Medicine, The Catholic University of Korea, Seoul St. Mary's Hospital, Seoul, Korea. ${ }^{2}$ Department of Hospital Pathology, Seoul St. Mary's Hospital, College of Medicine, The Catholic University of Korea, 222, Banpo-daero, Seocho-gu, Seoul 06591, Republic of Korea. ${ }^{3}$ Division of Gastrointestinal Surgery, Department of Surgery, Uijeongbu St. Mary's Hospital, College of Medicine, The Catholic University of Korea, Seoul, Korea, 271, Cheonbo-ro Uijeongbu, Gyeonggi-do 480-717, Republic of Korea. ${ }^{4}$ Department of Radiology, The Catholic University of Korea, Uijeongbu St. Mary's Hospital, Uijeongbu, Korea. ${ }^{5}$ Department of Radiology, The Catholic University of Korea, Seoul St. Mary's Hospital, Seoul, Korea. ${ }^{6}$ Department of Surgery, The Catholic University of Korea, Seoul St. Mary's Hospital, Seoul, Korea.
} 
Received: 31 May 2017 Accepted: 28 September 2017

Published online: 03 October 2017

\section{References}

1. Ferlay J, Soerjomataram I, Dikshit R, et al. Cancer incidence and mortality worldwide: sources, methods and major patterns in GLOBOCAN 2012. Int J Cancer. 2015;136(5):E359-86.

2. Mizoue T, Yoshimura T, Tokui N, et al. Prospective study of screening for stomach cancer in Japan. Int J Cancer. 2003;106(1):103-7.

3. Suh M, Choi KS, Lee $Y$ Y, et al. Cancer screening in Korea, 2012: results from the Korean National Cancer Screening Survey. Asian Pac J Cancer Prev. 2013;14(11):6459-63.

4. Isomoto H, Shikuwa S, Yamaguchi N, et al. Endoscopic submucosal dissection for early gastric cancer: a large-scale feasibility study. Gut. 2009; 58(3):331-6.

5. Gotoda T, Yanagisawa A, Sasako M, et al. Incidence of lymph node metastasis from early gastric cancer: estimation with a large number of cases at two large centers. Gastric Cancer. 2000;3(4):219-25.

6. Kunisaki C, Akiyama H, Nomura M, et al. Significance of long-term follow-up of early gastric cancer. Ann Surg Oncol. 2006;13(3):363-9.

7. Ye BD, Kim SG, Lee JY, et al. Predictive factors for lymph node metastasis and endoscopic treatment strategies for undifferentiated early gastric cancer. J Gastroenterol Hepatol. 2008;23(1):46-50.

8. Yamashita K, Sakuramoto S, Kikuchi S, et al. Validation of staging systems for gastric cancer. Gastric Cancer. 2008;11(2):111-8.

9. Kikuchi S, Futawatari N, Sakuramoto S, et al. Comparison of staging between the old (6th edition) and new (7th edition) TNM classifications in advanced gastric cancer. Anticancer Res. 2011;31(6):2361-5.

10. Nashimoto A, Akazawa K, Isobe Y, et al. Gastric cancer treated in 2002 in Japan: 2009 annual report of the JGCA nationwide registry. Gastric Cancer. 2013;16(1):1-27.

11. Lamouille $S, X u$ J, Derynck R. Molecular mechanisms of epithelialmesenchymal transition. Nat Rev Mol Cell Biol. 2014;15(3):178-96.

12. Thiery JP, Acloque $H$, Huang RY, et al. Epithelial-mesenchymal transitions in development and disease. Cell. 2009:139(5):871-90.

13. Kalluri $R$, Weinberg RA. The basics of epithelial-mesenchymal transition. J Clin Invest. 2009:119(6):1420-8.

14. Tam WL, Weinberg RA. The epigenetics of epithelial-mesenchymal plasticity in cancer. Nat Med. 2013;19(11):1438-49.

15. Barrallo-Gimeno A, Nieto MA. The Snail genes as inducers of cell movement and survival: implications in development and cancer. Development. 2005; 132(14):3151-61

16. Peinado $\mathrm{H}, \mathrm{Olmeda} \mathrm{D}, \mathrm{Cano} \mathrm{A}$. Snail, Zeb and bHLH factors in tumour progression: an alliance against the epithelial phenotype? Nat Rev Cancer. 2007;7(6):415-28.

17. Hajra KM, Chen DY, Fearon ER. The SLUG zinc-finger protein represses Ecadherin in breast cancer. Cancer Res. 2002;62(6):1613-8.

18. Bolos V, Peinado H, Perez-Moreno MA, et al. The transcription factor Slug represses E-cadherin expression and induces epithelial to mesenchymal transitions: a comparison with Snail and E47 repressors. J Cell Sci. 2003; 116(Pt 3):499-511.

19. Gupta PB, Kuperwasser C, Brunet JP, et al. The melanocyte differentiation program predisposes to metastasis after neoplastic transformation. Nat Genet. 2005;37(10):1047-54.

20. Phillips S, Kuperwasser CSLUG. Critical regulator of epithelial cell identity in breast development and cancer. Cell Adhes Migr. 2014;8(6):578-87.

21. Liu T, Zhang X, Shang M, et al. Dysregulated expression of Slug, vimentin, and E-cadherin correlates with poor clinical outcome in patients with basallike breast cancer. J Surg Oncol. 2013;107(2):188-94.

22. Hotz B, Arndt M, Dullat S, et al. Epithelial to mesenchymal transition: expression of the regulators snail, slug, and twist in pancreatic cancer. Clin Cancer Res. 2007;13(16):4769-76.

23. Uchikado $\mathrm{Y}$, Natsugoe $\mathrm{S}$, Okumura $\mathrm{H}$, et al. Slug Expression in the E-cadherin preserved tumors is related to prognosis in patients with esophageal squamous cell carcinoma. Clin Cancer Res. 2005:11(3):1174-80.

24. Storci G, Sansone P, Trere D, et al. The basal-like breast carcinoma phenotype is regulated by SLUG gene expression. J Pathol. 2008;214(1):25-37.

25. Yao C, Su L, Shan J, et al. IGF/STAT3/NANOG/Slug Signaling Axis Simultaneously Controls Epithelial-Mesenchymal Transition and Stemness Maintenance in Colorectal Cancer. Stem Cells. 2016;34(4):820-31.
26. Castro Alves C, Rosivatz E, Schott C, et al. Slug is overexpressed in gastric carcinomas and may act synergistically with SIP1 and Snail in the downregulation of E-cadherin. J Pathol. 2007;211(5):507-15.

27. Uchikado Y, Okumura H, Ishigami S, et al. Increased Slug and decreased Ecadherin expression is related to poor prognosis in patients with gastric cancer. Gastric Cancer. 2011;14(1):41-9.

28. Washington K. 7th edition of the AJCC cancer staging manual: stomach Ann Surg Oncol. 2010;17(12):3077-9.

29. Hu B, El Hajj N, Sittler S, et al. Gastric cancer: Classification, histology and application of molecular pathology. J Gastrointest Oncol. 2012;3(3):251-61.

30. Lauren P. The two histological main types of gastric carcinoma: diffuse and so-called intestinal-type carcinoma. An attempt at a Histo-clinical classification. Acta Pathol Microbiol Scand. 1965:64:31-49.

31. Yamashita K, Ooki A, Sakuramoto S, et al. Lymph node metastasis density (ND)-factor association with malignant degree and ND40 as "non-curative factor" in gastric cancer. Anticancer Res. 2008;28(1b):435-41.

32. Yamashita $\mathrm{K}$, Hosoda $\mathrm{K}$, Ema $\mathrm{A}$, et al. Lymph node ratio as a novel and simple prognostic factor in advanced gastric cancer. Eur J Surg Oncol. 2016; 42(9):1253-60.

33. Sano T, Aiko T. New Japanese classifications and treatment guidelines for gastric cancer: revision concepts and major revised points. Gastric Cancer. 2011:14(2):97-100

34. Roviello F, Rossi S, Marrelli D, et al. Number of lymph node metastases and its prognostic significance in early gastric cancer: a multicenter Italian study. J Surg Oncol. 2006;94(4):275-80. discussion 4

35. Pelz J, Merkel S, Horbach T, et al. Determination of nodal status and treatment in early gastric cancer. Eur J Surg Oncol. 2004;30(9):935-41.

36. Soetikno R, Kaltenbach $T$, Yeh $R$, et al. Endoscopic mucosal resection for early cancers of the upper gastrointestinal tract. J Clin Oncol. 2005;23(20): 4490-8.

37. Hyung WJ, Cheong JH, Kim J, et al. Application of minimally invasive treatment for early gastric cancer. J Surg Oncol. 2004;85(4):181-5. discussion 6

38. Kunisaki $C$, Takahashi $M$, Nagahori $Y$, et al. Risk factors for lymph node metastasis in histologically poorly differentiated type early gastric cancer. Endoscopy. 2009:41(6):498-503.

39. Barber ME, Save V, Carneiro F, et al. Histopathological and molecular analysis of gastrectomy specimens from hereditary diffuse gastric cancer patients has implications for endoscopic surveillance of individuals at risk. Pathol. 2008:216(3):286-94.

\section{Submit your next manuscript to BioMed Central and we will help you at every step:}

- We accept pre-submission inquiries

- Our selector tool helps you to find the most relevant journal

- We provide round the clock customer support

- Convenient online submission

- Thorough peer review

- Inclusion in PubMed and all major indexing services

- Maximum visibility for your research

Submit your manuscript at www.biomedcentral.com/submit 